\title{
Evolutionary forces on Anopheles: what makes a malaria vector?
}

\author{
Anna Cohuet ${ }^{1}$, Caroline Harris, Vincent Robert and Didier Fontenille
}

Institut de Recherche pour le Développement, UR016, “Characterization and control of vector populations”, 911 avenue Agropolis, BP 64501, 34394 Montpellier cedex 5, France

\begin{abstract}
In human malaria, transmission intensity is highly dependent on the vectorial capacity and competence of local mosquitoes. Most mosquitoes are dead ends for the parasite, and only limited ranges of Anopheles are able to transmit Plasmodium to humans. Research to understand the determinants of vectorial capacity and competence has greatly progressed in recent years; however, some aspects have been overlooked and the evolutionary pressures that affect them often neglected. Here, we review key factors of vectorial capacity and competence in Anopheles, with a particular focus on the most important malaria vector Anopheles gambiae. We aim to point out selection pressures exerted by Plasmodium on Anopheles to improve its own transmission and discuss how the parasite might shape the vector to its benefit.
\end{abstract}

\section{Vectorial capacity and competence in malaria transmission}

The vectorial capacity of a mosquito population largely determines the intensity of vector-borne disease transmission (Box 1). The vector competence (Glossary) is also a crucial parameter for the pathogen to be transmitted. In human malaria, vectorial systems are limited in number. Only Anopheles females are able to transmit Plasmodium to humans, and, among the more than 450 Anopheles species known, $\sim 60$ are considered to be actual vectors in the wild [1]. Vectorial capacity and competence also present quantitative features in the sense that some species have a major role in malaria transmission, and others have a minor role. Even at the species level, some populations or individual mosquitoes can have different impacts on transmission [1]. Research to understand the genetic determinants of capacity and competence has greatly benefited from the availability of the whole genome sequence for Anopheles gambiae [2] with the identification of candidate genes in progress. However, the different aspects of vectorial capacity and competence have not been uniformly studied, and some have been largely overlooked. For example, rapid progression has been recently made in mosquito immunity and olfaction genetics [3-5]; however, genetic determinants of parasite virulence and mosquito adaptation to human environments remain minor research areas. Moreover, evolutionary pressures on vectors, including the forces exerted by the parasites they transmit,

\footnotetext{
Corresponding author: Cohuet, A. (anna.cohuet@ird.fr)

${ }^{1}$ Current address: Institut de Recherche en Sciences de la Santé, 399, Avenue de la Liberté, 01 BP 545 Bobo-Dioulasso 01, Burkina Faso.
}

can have major consequences on malaria transmission and are rarely considered for their impact on malaria control measures. Here, we address the major aspects of vectorial capacity and competence and the evolutionary forces that affect them in Anopheles: the vector longevity; the duration of sporogonic development; the contact between the mosquito and vertebrate host suitable for the parasite; and the susceptibility/resistance of the vector to the parasite. We aim to point out selection pressures exerted by Plasmodium on malaria vectors to improve transmission, particularly in the Plasmodium falciparum-An. gambiae system, and discuss their relevance in malaria control.

\section{Vector longevity}

Malaria parasite development in vector mosquitoes requires passing through two epithelia and results in thousands of parasites [6]. The sporogonic development might therefore impose some degree of virulence (Glossary) and affect the fitness (Glossary) of the vector host. The infection-induced fitness cost can be expressed as a reduction of survival or fecundity [7] but an effect on survival would have a much higher impact on malaria transmission (Box 1) as the vector must live long enough to become infectious. Several mechanisms of Plasmodium virulence to mosquito vectors can be expected. Some were tested but mainly in experimental Plasmodium-Anopheles systems. The results on reduction of longevity are conflicting with many studies showing vector survival to be unaffected by infection, but some showing the opposite [8] Potential mechanisms of virulence are: (i) the passage of parasites through the mosquito epithelia that can cause cell damage. It was observed that Plasmodium sp. causes

\section{Glossary}

Anthropophilic behavior: mosquito preference for breeding sites created by human activities, using human dwellings as resting places and humans as a source of bloodmeal.

Fitness: here, we consider it to be the success of individuals or populations in producing offspring, relative to others.

Refractoriness: intrinsic ability of Anopheline species or populations to not replicate and not transmit different Plasmodium species (or strains).

Resistance: mechanisms of host defense responsible for reducing parasite burden, it includes the immune response.

Susceptibility: intrinsic ability of Anopheline species or populations to replicate and transmit different Plasmodium species (or strains). It is approximated by experimental transmissions.

Tolerance: in tolerance, the host fights the disease by repairing damage caused by the infection, for example tissue repair.

Vector competence: most authors consider that it is synonymous to susceptibility.

Virulence: here, it is interpreted as the negative effects caused by the parasite on the mosquito in terms of survival or fecundity. 


\section{Box 1. Vectorial capacity and basic reproductive rate}

Vectorial capacity is defined as 'the average number of inoculations with a specified parasite, originating from one case of malaria in unit time that the population would distribute if all the vector females biting the case become infected'. It can be summarized by the Macdonald formula [61].

$C:\left(m a^{2} p^{n} /-\ln p\right)$

$\boldsymbol{m}$ is the vector-host ratio (i.e. the anopheline density in relation to humans). $a$ is the human feeding rate: the number of human bites per mosquito, per day. $\boldsymbol{p}$ is the daily survival rate (i.e. the probability of a mosquito surviving one whole day). In $\boldsymbol{p}$ is the natural logarithm of $\boldsymbol{p}$. The mosquito longevity is $1 / p . n$ is the number of days required for sporogonic development (i.e. the time necessary for parasites to complete development from ingested gametocytes in the bloodmeal to sporozoites in the salivary glands).

The basic reproductive rate $\left(R_{0}\right)$ is the total number of malaria cases derived from one infective case that the mosquito population would distribute to man. $R_{0}$ must equal at least 1 for the disease to persist or spread. For values less than 1, the disease will regress. It is the product of the vectorial capacity, the infectiousness of vectors to humans $(\boldsymbol{b})$ and humans to vectors $(\boldsymbol{c})$, and the human infectious period $(1 / r)$ :

$R_{0}=\left(m a^{2} p^{n} /-\ln p\right) * b c * 1 / r$

$\mathrm{c}$ is closely linked to the vector competence of mosquitoes to Plasmodium species.

In $R_{0}$, two parameters are more important than the others: ' $a$ ' and ' $p$ '. ' $a$ ', the human feeding rate is squared because the mosquito needs to bite twice to transmit the parasite, firstly to become infected, and secondly to infect, after completion of sporogonic development. For this reason, a small change in mosquito feeding preference will have a serious impact on malaria transmission. Even more importantly, ' $p$ ', the survival rate, owing to its power $n$, has a disproportionate impact on transmission. As the sporogonic development of Plasmodium is relatively long (e.g. for $P$. falciparum: 12 23 days [30]) in relation to mosquito lifespan (2-4 weeks for $A n$. gambiae [1]), mosquitoes can be infectious for a limited period only. Small changes in vector longevity can dramatically affect malaria transmission [62]. Modeling highlights that longevity is the factor with the biggest impact on the basic reproductive rate $\left(R_{0}\right)$, even more so than initially suggested by Macdonald [61].

host cell damage, followed by apoptosis [9,10]; however, the effect of infection-induced apoptosis on mosquito survival remains unclear. Programmed cell death was observed in the midgut cells but also in the follicular epithelial cells in the ovaries, resulting in reduced egg production. The cell damage could therefore impact fecundity rather than survival [11]. (ii) The infection generates a mosquito immune response that can be costly. Parasite invasion of mosquito vectors in model and natural systems induces a massive immune response $[12,13]$ and several studies in insects demonstrate that such an induced response can affect fecundity [14] or longevity [15]. In Anopheles, a reproductive cost was found [16] and An. gambiae mosquito strains selected for refractoriness to rodent parasites show reduced fitness compared with susceptible strains $[17,18]$. This suggests that developing an immune response induces a fitness cost, but more research is needed to establish whether there is an effect on mosquito survival. (iii) Competition for energy resources between the host and the developing parasites can have a negative impact on the host. In agreement with this hypothesis, a reduced survival was observed in Anopheles stephensi infected with rodent parasites and was most striking under the condition of glucose deprivation [19]; moreover, infection increases mosquito sugar feeding [20]. (iv) Another potential cost of infection is that the parasite can affect mosquito behavior and cause mortality. In the natural An. gambiae- $P$. falciparum system, a higher feeding associated mortality was observed, probably as a result of a decreased efficiency in bloodmeal intake, and increased feeding activity [21]. This is, in fact, the only effect of infection on mosquito survival to be observed in this natural system. It could be the result of compensatory behavior by the mosquito to obtain sufficient blood for its trophogonic cycle, or a manipulation of the behavior of the mosquito by the parasite to increase its transmission by multiplying mosquito blood feeds [22]. Exploiting compensatory behavior can be considered a particularly well-developed strategy of manipulation by the parasite [23].

Therefore, in spite of the potentially high virulence of Plasmodium to mosquito vectors, an effect on vector longevity is not always observed. The reduction of fecundity is a more common cost of infection and has only a minor effect on transmission. It is likely that evolutionary forces selected parasites affecting mosquito fecundity rather than that affecting mosquito survival, and this eventually benefited parasite transmission [11]. Moreover, meta-analysis of several published studies demonstrated that infectioninduced mortality is less likely to be found in natural systems than in experimental vector-parasite species combinations [8], which could be explained by evolutionary forces acting between coevolved parasites and hosts. As both vector and parasite share the interest of vector survival, parasites might evolve towards low virulence, whereas, in parallel, mosquitoes less affected by infection would be selectively advantaged. For this to be true, virulence in Plasmodium-Anopheles interactions must have a genetic basis; this has been shown in some experiments, with both parasite and mosquito genetics affecting infection success [24-26]. However, the evolution of virulence is also highly dependent on environmental factors [19,24,25]. The fact that $P$. falciparum and An. gambiae share an evolutionary history, by contrast to laboratory model systems, might have led to the selection of mechanisms in both the parasite and the mosquito to reduce any negative impact on host survival and subsequently increase the chances of parasite transmission.

Recent studies emphasize the concept of tolerance (Glossary) that might play a role in host defense against the parasite in parallel to resistance (Glossary). Tolerance is expected to be costly and could also impact survival or fecundity as the host spends resources to repair any damage the infection has caused. But, unlike resistance, tolerance to the effects of disease-induced mortality has a positive effect on parasite development $[27,28]$ and could be an important mechanism in Plasmodium-Anopheles interactions. This opens a new avenue of research in the malaria vectorial system.

Today, there is a crucial need to decipher parasite effects on mosquito fitness, the mechanisms involved and their genetic and environmental determinants to predict how parasite virulence evolves. This is particularly relevant for the prospect of using genetically modified mosquitoes (GMMs) to control malaria transmission. A recent study showed that the cost of genetic transformation 


\section{Box 2. Vector longevity, insecticide resistance and malaria transmission control}

Insecticides reduce mosquito longevity, the most important parameter of vectorial capacity. However, insecticide resistance limits the efficacy of vector-control measures and can interact with the parasite. New strategies could limit the emergence of resistance.

\section{Selection pressures for insecticide resistance in malaria control} The use of insecticides for agriculture or public health results in a strong selection pressure [63]. In malaria vectors, multiple resistance mechanisms appeared independently and/or were able to spread in spite of strong gene flow barriers. For instance, several mutation events were selected independently in An. gambiae [64], and through introgression were passed to other members of the Gambiae complex [65].

\section{Effect of insecticide resistance on infection cost}

Genetic insecticide resistance was shown to impact the infection level in the invertebrate host. For instance, several studies showed that insecticide resistant Culex were more heavily infected by Wolbachia and suffered higher infection costs [66]. By contrast, in the case of filarial infection, insecticide resistant mosquitoes were less infected than susceptible ones. Insecticide resistance might affect parasite transmission in mosquitoes by changing potential redox reactions in several tissues and in doing so it was proposed that it could provide direct protection against infection [67]. To our knowledge, no studies have been published on the relationship between insecticide resistance in Anopheles and infection level/cost of Plasmodium, although they would be highly relevant to malaria control.

Killing mosquitoes responsible for malaria transmission and limiting insecticide resistance

Today, new elegant strategies are emerging to reduce vector longevity and subsequently reduce malaria transmission [68]. One concept is to use late-life acting (LLA) insecticides that would target older mosquitoes. The benefit of such a strategy is that it kills mosquitoes before they become infectious but after the majority of mosquito reproduction has taken place, limiting the selective pressure for resistance mechanisms. The LLA insecticides aim to kill the mosquito some time after exposure or target physiologically vulnerable mosquitoes based on the logic that older mosquitoes will be more sensitive. Insecticides with a new mode of action or new mode of administration are needed. Knowledge on mechanisms of senescence in mosquitoes or on the effects of pathogens could allow the development of alternative strategies such as the use of pathogens (e.g. Wolbachia or fungal pathogens [69]) with potentially synergistic effects with Plasmodium or insecticides on survival reduction. could be compensated for by the ability to resist infection in a laboratory model [29]. The potential success of the GMM strategy in the wild will be highly dependent on the balance between this cost and benefit to the mosquito, an area closely linked to the prevalence and virulence of infection. Moreover, vector longevity is likely to become a major target to control malaria transmission, for which we need a better understanding of the interactions between vectors, pathogens and insecticides in natural conditions (Box 2).

\section{Incubation time of the parasite in the vector}

The duration of sporogonic development is also a crucial factor in vectorial capacity. The earlier the sporozoites reach the salivary glands, the greater the opportunity to be transmitted; this therefore exerts a strong selective pressure to reduce the duration of sporogonic development. This assumption contradicts what we observe, as the development time in the vector is relatively long for Plasmodium (12-23 days [30]). Koella [22] hypothesized that this long duration of sporogonic development is necessary to produce a high number of sporozoites. Such a high number of parasites disturbs the efficacy of blood feeding and induces a higher biting rate and thus could be responsible for vector behavior manipulation [22]. In terms of transmission strategy, the parasite could be transmitted (i) as early as possible or (ii) later, when numbers of sporozoites are higher. As we observe this higher biting rate in infectious mosquitoes and a long sporogonic development time, it seems that the second strategy was selected. A higher biting rate comes with a greater risk of death and is likely to infer a manipulation cost on mosquito survival; this could, however, be compensated for by the benefit to the parasite in increasing its transmission.

A method to optimize the parasites adaptive interest in developing high numbers of sporozoites and shortening their development time is to carry out the cycle at its optimal temperature. It can be hypothesized that the parasite manipulates its vector in this way to increase transmission. Indeed, the sporogony dynamics are dependent on temperature; with a species-specific threshold, temperature is negatively correlated with development time [30]. A parasite-induced behavioral modification has been shown in $P$. mexicanum-infected phlebotomine sand flies, making them more attracted to higher temperatures than are non-infected individuals [31]. This suggests that Plasmodium might be capable of manipulating mosquito temperature preferences to increase their vectorial capacity, which warrants investigation in the natural human malaria system.

\section{Human-mosquito contact and human biting rate}

The density of vectors in contact with humans and the vertebrate host preference for mosquito bloodmeals are closely related. The anthropophilic behavior (Glossary) of An. gambiae is an important factor in its high vectorial capacity (Box 1). The hypothesis developed by Coluzzi explains the enhanced contact between humans and $A n$. gambiae some thousands of years ago and the subsequent drastic changes in vectorial capacity [32]. An extensive penetration of forests began $\sim 3000$ years ago by Bantu populations, which have established agriculture through deforestation [33]. The An. gambiae ancestors, previously not able to survive in forests, could then find suitable sunny breeding sites and invade this new ecological niche. In parallel, a strong selection pressure against cattle, owing to trypanosomiasis, had the consequence that humans were the most frequent large vertebrate hosts available in such areas [32]. By providing the breeding sites and the bloodmeal to the newly arrived Anopheles, humans served as 'board and lodging', and selected the highly specialized species, An. gambiae, whose biology became very dependent on humans. This specialization to humans has been differentially selected in the members of the An. gambiae complex [32]. The adaptation of these different species to diverse environments and their related trophic behavior 
was accompanied by the fixation of different chromosomal arrangements, which are known to protect coadapted alleles from recombination [34]. The association between chromosomal inversions and host preference provides evidence of a genetic basis for trophic behavior [35] and makes it susceptible to selective forces. The rapid adaptation of An. gambiae s.s. to humans and the specialization of the members of the complex to diverse environments is a clear illustration of their genetic diversity and plasticity.

The adaptation of An. gambiae ancestors to humans was accompanied by a dramatic increase in $P$. falciparum transmission. The traditional view of the story highlights the benefit of vector adaptation to its vertebrate host, but it could also be as a result of selection pressures exerted by the parasite to increase its transmission that might have strengthened the specialization. Several experiments revealed the ability of Plasmodium to modify the trophic behavior of the mosquito host. Vectors show a preference for biting gametocyte-infected human hosts [36] and pregnant women (who are generally more heavily infected) [37], and infected vectors are more aggressive $[38,39]$. One might think that the proportion of infected mosquitoes in nature would not allow strong selection pressure to be exerted by the parasite on mosquito behavior. However, considering the daily mortality of An. gambiae (estimated at $10-18 \%$ [40]) and the long sporogonic development (1014 days), the $5 \%$ sporozoite infection rate frequently observed [41] means that a large proportion of An. gambiae are in contact with the parasite during their lifespan, and suggests that trophic behavior might be under selective pressures to increase parasite transmission [42].

Genetic determinants of adaptation to human environments and trophic behavior of malaria vectors remain almost unknown. Current investigation is based on the assumption that olfaction has a crucial role in behavior, at least in mosquito host choice for a particular bloodmeal. Recent descriptions of cellular and molecular olfactory components $[4,5]$ open promising avenues for discovering how mosquitoes choose their vertebrate host for a bloodmeal and therefore the potential to modify its trophic behavior to limit malaria transmission.

\section{Susceptibility and refractoriness to infection}

A mosquito can be considered as a competent malaria vector if the Plasmodium parasite can internally complete its cycle from gametocytes in the bloodmeal to sporozoites in the saliva. We can distinguish different levels of susceptibility (Glossary) for human malaria parasites: a qualitative level with complete refractoriness (Glossary) of non-Anopheles species and a quantitative level among Anopheles species.

Numerous culicidae are in close contact with humans and can, in some cases, transmit infectious agents to them. Nevertheless, to our knowledge, no reports show human malaria parasite development in mosquitoes other than Anopheles. The density and the human biting rates of nonmalaria vector mosquitoes can be high in areas of intense malaria transmission. Therefore, the ingestion of $P$. falciparum by non-vector species must be frequent; however, the vector competence has not been selected in a wide range of species. This suggests that crucial factors avoiding infection in non-competent mosquito species must be highly differentiated from competent ones. The study of early parasite developmental steps in non-malaria vectors could therefore be used to point out essential mosquito factors in Anopheles-Plasmodium interactions and shed light on new targets for transmission blocking strategies.

In Anopheles, the susceptibility to human Plasmodium parasites appears to be more quantitative. Experimental infections of Anopheles species that do not naturally transmit $P$. falciparum often show low levels of infection but rarely none $[43,44]$. The outcome of infection depends on the mosquito response and the ability of the parasite to evade it. Indeed, the presence of infectious agents in the mosquito is considered as non-self and induces an immune response that can limit the infection efficiency [45]. Extensive work, mainly using model parasite-vector systems, partially deciphered the An. gambiae immune system $[46,47]$. Today, more effort is invested in research on natural vector-parasite systems. Genes playing a role in the mosquito response to $P$. falciparum have been identified [12,13]; a genetic island of resistance was mapped demonstrating that genetic variants play a role in susceptibility and resistance in mosquito vectors, and a candidate gene encoding a leucine-rich protein (APL1) shows alleledependant resistance [48,49]. Comparisons of different vector-parasite combinations revealed that parts of the mosquito response mechanism are general and effective against several infectious agents [12,13], whereas others are more specific $[13,50]$. For instance, melanization appeared to be a baseline response to Plasmodium infection or other foreign bodies (e.g. sephadex beads) in An. gambiae [51] as in other mosquito species [43]. However, in the case of An. gambiae infected by $P$. falciparum, melanization does not appear as the major infection control mechanism, although it is occasionally observed in experimentally infected mosquitoes [48,52]. Also, silencing of key melanization genes did not affect the level of infection by $P$. falciparum: this contrasts with what was obtained when infected by $P$. berghei [50]. Thus, the mechanism of melanization appears to be a highly efficient mosquito response against non-self, except against the coadapted human malaria parasite. This suggests that Plasmodium either suppresses the immune response or develops evasion mechanisms [51,53]. The fact that key immune related mosquito genes are upregulated during $P$. falciparum infection $[12,13,50]$ favors the hypothesis of evasion. Its capabilities to evade the response could be a major factor in vector competence.

The evolutionary arms race between parasites and hosts predicts constant evolution of mosquito refractoriness and parasite evasion mechanisms. The high diversity and plasticity of Plasmodium in vertebrate hosts leads to the assumption that the parasite evolves faster, and adapts more rapidly, than the host, and is expected to transmit more successfully through a local coevolved vector population, rather than a non-local one. In other words, the parasite would be the fastest runner in the host-parasite race. In agreement, several European Anopheles species that were tested for vector competence for $P$. falciparum showed the development of low numbers of parasites compared with An. gambiae [44]. These European species are 
therefore competent with low efficiency for $P$. falciparum transmission. Thus, the high vector competence of $A n$. gambiae could be partially because of the fact that African $P$. falciparum is well adapted to this vector, and the frequency and intensity of contacts between the parasite and the mosquito have determined the level of coadaptation. This would explain the efficient transmission of $P$. falciparum by its local vectors, but predicts that an introduction of $P$. falciparum in areas lacking this parasite, where vector populations are not coadapted, would not rapidly lead to a high level of transmission. However, it cannot be neglected that new contacts between $P$. falciparum and Anopheles are sometimes efficient: studies carried out in non-natural host-parasite combinations sometimes reveal high vector susceptibility [54,55]. It would be of great interest to be able to predict the success of new Anopheles-Plasmodium interactions in the context of drastic environmental changes such as current global warming.

The outcome of infection is parasite genotype-vector genotype dependant; no parasite has optimal transmission in all hosts and no host resists all parasites. As shown by Lambrechts et al. [56], An. gambiae isofemale lines can be highly susceptible to some parasite genotypes, and, at the same time, highly resistant to others. Furthermore, Plasmodium infectivity and mosquito susceptibility are environment-dependant [57]. Such genotype-genotypeenvironment interactions occur when one parasite genotype is more successful (better transmission) with one host genotype and less with another, but these interactions change when the host and parasite are exposed to different environments [58] and are known to affect insect immunity [59]. The mosquito genome has therefore been thoroughly searched for genetic determinants of vector susceptibility or refractoriness, although parasite genetics could be as important. Moreover, the efficiency of potential mosquito resistance genes in natural situations might differ depending on environment and parasite genetics [60]. Studies on the effect of the environment and parasite diversity on the evolution of vector competence are crucial in understanding Anopheles-Plasmodium interactions and identifying suitable targets for malaria control.

\section{Concluding remarks}

Vectorial capacity and competence in the malaria system is the result of constant evolution of mosquitoes in relation to the other evolving members of the system (parasites and humans) and a changing environment (Figure 1). Here, we have outlined the role of the parasite on the evolution of vectorial capacity and competence. The parasite is likely to exert selection pressures on the vector to shape it to its benefit, such as it having a sufficient longevity, a specialization for feeding on humans and an immune system that it can evade. From this point of view, $P$. falciparum was extremely successful in selecting its main vector, $A n$. gambiae. On other vectors, $P$. falciparum probably exerted lower selection pressures as a result of less frequent contacts between them, giving those vectors a less important role in malaria transmission. That is the case for the phylogenetically related species of the Gambiae complex to An. gambiae s.s., a situation similar to that of most other species complexes. Studies on molecular evolution and the comparison of genomes between closely related species with differences in vectorial capacity and competence will shed light on selective forces specific to vectors. We expect strong evolutionary forces on genes involved in the most important parameters of vectorial capacity (longevity, host choice) and, maybe, lower selective pressure on others (vector immunity). Such studies could help to find new targets for malaria control. We currently face the great challenge of identifying genetic and environmental components of vectorial capacity and competence. Field studies

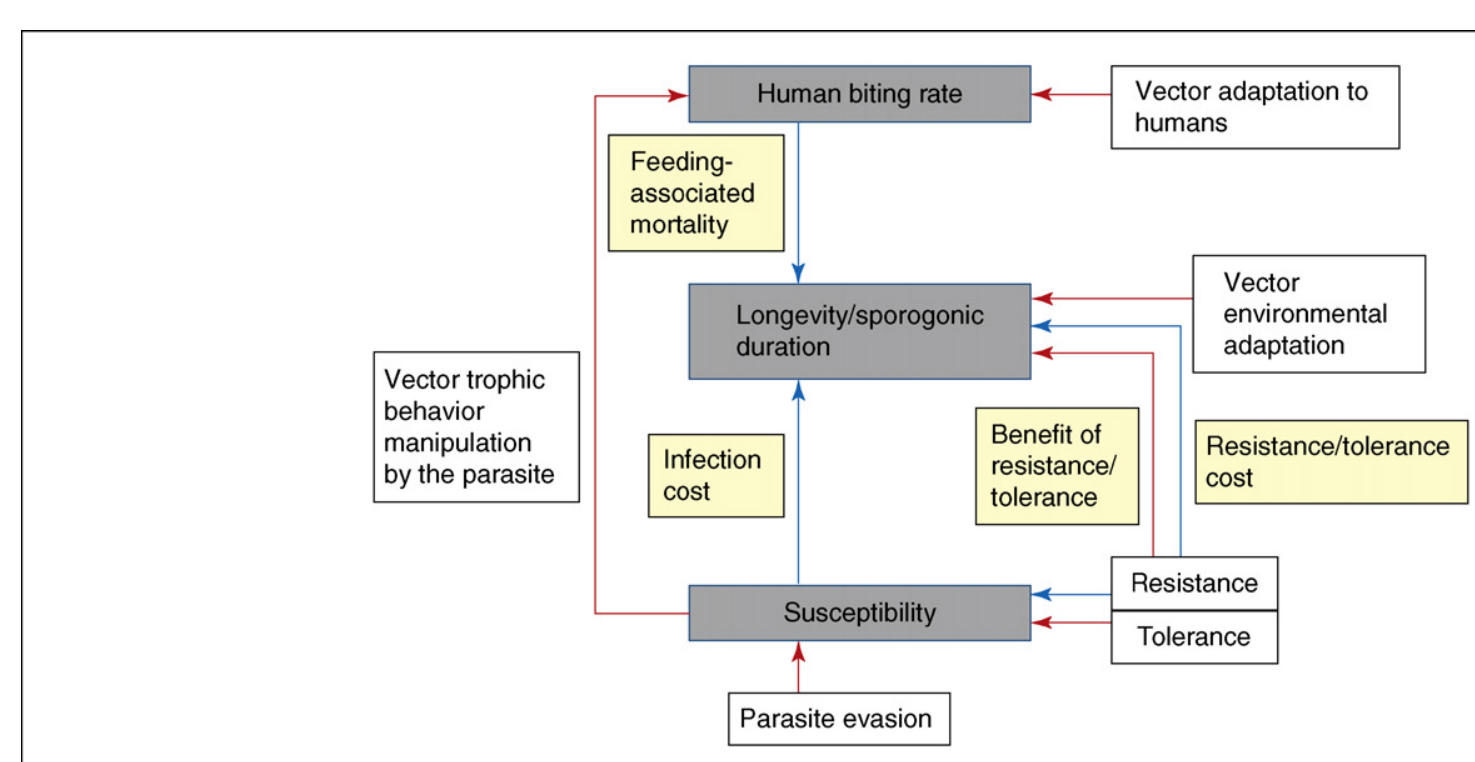

$\overline{\text { TRENDS in Parasitology }}$

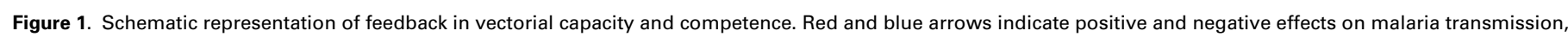

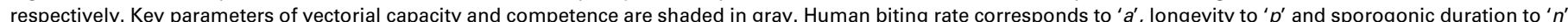

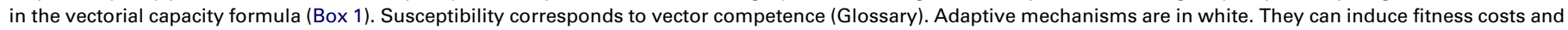
benefits to the parasite or the vector (shaded in yellow) and have positive or negative impacts on transmission. 
must be multiplied and take into account the evolutionary forces exerted in the wild, particularly by the parasite. Considering the evolutionary forces that affect vectorial capacity and competence is important in determining the epidemiology of malaria, and considering evolutionary ecology in the development of control strategies appears essential.

\section{Acknowledgements}

We are grateful to Frédéric Simard and Frédéric Thomas for fruitful discussions, and to Steven M. Goodman for commenting on an earlier draft of this paper. We acknowledge five anonymous reviewers for constructive suggestions. This work was funded by an Agence Nationale de la Recherche, programme "Microbiologie Immunologie et Maladies Emergentes" (ANR MIME) grant to A.C. and C.H.

\section{References}

1 Manguin, S. et al. (2008) Biodiversity of Malaria in the World, John Libbey Eurotext

2 Holt, R.A. et al. (2002) The genome sequence of the malaria mosquito Anopheles gambiae. Science 298, 129-149

3 Christophides, G.K. et al. (2002) Immunity-related genes and gene families in Anopheles gambiae. Science 298, 159-165

$4 \mathrm{Lu}$, T. et al. (2007) Odor coding in the maxillary palp of the malaria vector mosquito Anopheles gambiae. Curr. Biol. 17, 1533-1544

5 Hallem, E.A. et al. (2006) Insect odor and taste receptors. Annu. Rev. Entomol. 51, 113-135

6 Vaughan, J.A. (2007) Population dynamics of Plasmodium sporogony. Trends Parasitol. 23, 63-70

7 Ferguson, H. et al. (2006) Malaria parasite virulence in mosquitoes and its implications for the introduction and efficacy of GMM malaria control programmes. In Genetically Modified Mosquitoes for Malaria Control (Boëte, C., ed.), pp. 103-116, Landes Bioscience

8 Ferguson, H.M. and Read, A.F. (2002) Why is the effect of malaria parasites on mosquito survival still unresolved? Trends Parasitol. 18, $256-261$

9 Hurd, H. and Carter, V. (2004) The role of programmed cell death in Plasmodium-mosquito interactions. Int. J. Parasitol. 34, 1459-1472

10 Vlachou, D. et al. (2004) Real-time, in vivo analysis of malaria ookinete locomotion and mosquito midgut invasion. Cell Microbiol. 6, 671-685

11 Hurd, H. et al. (2005) Interactions between malaria and mosquitoes: the role of apoptosis in parasite establishment and vector response to infection. Curr. Top. Microbiol. Immunol. 289, 185-217

12 Mendes, A.M. et al. (2008) Conserved mosquito/parasite interactions affect development of Plasmodium falciparum in Africa. PLoS Pathog. 4, e1000069

13 Dong, Y. et al. (2006) Anopheles gambiae immune responses to human and rodent Plasmodium parasite species. PLoS Pathog. 2, e52

14 Schwartz, A. and Koella, J.C. (2004) The cost of immunity in the yellow fever mosquito, Aedes aegypti depends on immune activation. J. Evol. Biol. 17, 834-840

15 Armitage, S.A. et al. (2003) Examining costs of induced and constitutive immune investment in Tenebrio molitor. J. Evol. Biol. $16,1038-1044$

16 Ahmed, A.M. and Hurd, H. (2006) Immune stimulation and malaria infection impose reproductive costs in Anopheles gambiae via follicular apoptosis. Microbes Infect. 8, 308-315

17 Voordouw, M.J. et al. (2009) Rodent malaria-resistant strains of the mosquito, Anopheles gambiae, have slower population growth than susceptible strains. BMC Evol. Biol. 9, 76

18 Hurd, H. et al. (2005) Evaluating the costs of mosquito resistance to malaria parasites. Evolution 59, 2560-2572

19 Lambrechts, L. et al. (2006) Environmental influence on the genetic basis of mosquito resistance to malaria parasites. Proc. Biol. Sci. 273, 1501-1506

20 Rivero, A. and Ferguson, H.M. (2003) The energetic budget of Anopheles stephensi infected with Plasmodium chabaudi: is energy depletion a mechanism for virulence? Proc. Biol. Sci. 270, 1365-1371

21 Anderson, R.A. et al. (2000) Plasmodium falciparum sporozoites increase feeding-associated mortality of their mosquito hosts Anopheles gambiae s.l. Parasitology 120, 329-333
22 Koella, J.C. (1999) An evolutionary view of the interactions between anopheline mosquitoes and malaria parasites. Microbes Infect. 1, 303308

23 Lefevre, T. et al. (2008) Exploiting host compensatory responses: the 'must' of manipulation? Trends Parasitol. 24, 435-439

24 Ferguson, H.M. et al. (2003) Mosquito mortality and the evolution of malaria virulence. Evolution 57, 2792-2804

25 Ferguson, H.M. and Read, A.F. (2002) Genetic and environmental determinants of malaria parasite virulence in mosquitoes. Proc. Biol. Sci. 269, 1217-1224

26 Ferguson, H.M. et al. (2003) The influence of malaria parasite genetic diversity and anaemia on mosquito feeding and fecundity. Parasitology 127, 9-19

27 Best, A. et al. (2008) Maintenance of host variation in tolerance to pathogens and parasites. Proc. Natl. Acad. Sci. U.S.A. 105, 2078620791

28 Read, A.F. et al. (2008) Animal defenses against infectious agents: is damage control more important than pathogen control. PLoS Biol. 6 (12), e4

29 Marrelli, M.T. et al. (2007) Transgenic malaria-resistant mosquitoes have a fitness advantage when feeding on Plasmodium-infected blood. Proc. Natl. Acad. Sci. U.S.A. 104, 5580-5583

30 World Health Organization (1975) WHO Manual on Practical Entomology in Malaria, Part II, World Health Organization

31 Fialho, R.F. and Schall, J.J. (1995) Thermal ecology of a malarial parasite and its insect vector: consequences for the parasite's transmission success. J. Anim. Ecol. 64, 553-562

32 Ayala, F.J. and Coluzzi, M. (2005) Chromosome speciation: humans, Drosophila, and mosquitoes. Proc. Natl. Acad. Sci. U.S.A. 102 (Suppl. 1), 6535-6542

33 Willis, K.J. et al. (2004) Ecology. How 'virgin' is virgin rainforest? Science 304, 402-403

34 Coluzzi, M. (1982) Spatial distribution of chromosomal inversions and speciation in Anopheline mosquitoes. Prog. Clin. Biol. Res. 96, 143-153

35 Takken, W. and Costantini, C. (2006) The genetics of vector-host interactions. In Genetically Modified Mosquitoes for Malaria Control (Boëte, C., ed.), pp. 137-145, Landes Bioscience

36 Lacroix, R. et al. (2005) Malaria infection increases attractiveness of humans to mosquitoes. PLoS Biol. 3, e298

37 Ansell, J. et al. (2002) Short-range attractiveness of pregnant women to Anopheles gambiae mosquitoes. Trans. R. Soc. Trop. Med. Hyg. 96, 113-116

38 Wekesa, J.W. et al. (1992) Effect of Plasmodium falciparum on blood feeding behavior of naturally infected Anopheles mosquitoes in western Kenya. Am. J. Trop. Med. Hyg. 47, 484-488

39 Koella, J.C. et al. (1998) The malaria parasite, Plasmodium falciparum, increases the frequency of multiple feeding of its mosquito vector, Anopheles gambiae. Proc. Biol. Sci. 265, 763-768

40 Costantini, C. et al. (1996) Density, survival and dispersal of Anopheles gambiae complex mosquitoes in a west African Sudan savanna village. Med. Vet. Entomol. 10, 203-219

41 Bass, C. et al. (2008) PCR-based detection of Plasmodium in Anopheles mosquitoes: a comparison of a new high-throughput assay with existing methods. Malar. J. 7, 177

42 Prugnolle, F. et al. (2009) Infection and body odours: evolutionary and medical perspectives. Infect. Genet. Evol. 9, 1006-1009

43 Habtewold, T. et al. (2008) Transmission blocking immunity in the malaria non-vector mosquito Anopheles quadriannulatus species A. PLoS Pathog. 4, e1000070

44 de Zulueta, J. et al. (1975) Receptivity to malaria in Europe. Bull World Health Organ. 52 (1), 109-111

45 Sinden, R.E. et al. (2004) Mosquito - malaria interactions: a reappraisal of the concepts of susceptibility and refractoriness. Insect. Biochem. Mol. Biol. 34, 625-629

46 Blandin, S.A. et al. (2009) Dissecting the genetic basis of resistance to malaria parasites in Anopheles gambiae. Science 326, 147-150

47 Povelones, M. et al. (2009) Leucine-rich repeat protein complex activates mosquito complement in defense against Plasmodium parasites. Science $324,258-261$

48 Riehle, M.M. et al. (2006) Natural malaria infection in Anopheles gambiae is regulated by a single genomic control region. Science $312,577-579$ 
49 Mitri, C. et al. (2009) Fine pathogen discrimination within the APL1 gene family protects Anopheles gambiae against human and rodent malaria species. PLoS Pathog. 5, e1000576

50 Cohuet, A. et al. (2006) Anopheles and Plasmodium: from laboratory models to natural systems in the field. EMBO Rep. 7, 12851289

51 Lambrechts, L. et al. (2007) Effect of infection by Plasmodium falciparum on the melanization immune response of Anopheles gambiae. Am. J. Trop. Med. Hyg. 76, 475-480

52 Schwartz, A. and Koella, J.C. (2002) Melanization of Plasmodium falciparum and C-25 sephadex beads by field-caught Anopheles gambiae (Diptera: Culicidae) from southern Tanzania. J. Med. Entomol. 39, 84-88

53 Boete, C. et al. (2004) Direct and indirect immunosuppression by a malaria parasite in its mosquito vector. Proc. Biol. Sci. 271, $1611-1615$

54 Collins, W.E. and Roberts, J.M. (1991) Anopheles gambiae as a host for geographic isolates of Plasmodium vivax. J. Am. Mosq. Control Assoc. $7,569-573$

55 Nace, D. et al. (2004) Susceptibility of Anopheles farauti to infection with different species of Plasmodium. J. Am. Mosq. Control Assoc. 20, $272-276$

56 Lambrechts, L. et al. (2005) Host genotype by parasite genotype interactions underlying the resistance of anopheline mosquitoes to Plasmodium falciparum. Malar. J. 4, 3

57 Tripet, F. et al. (2008) Ecological immunology of mosquito-malaria interactions. Trends Parasitol. 24, 219-227

58 Wolinska, J. and King, K.C. (2009) Environment can alter selection in host-parasite interactions. Trends Parasitol. 25, 236-244
59 Lazzaro, B.P. et al. (2008) Genotype-by-environment interactions and adaptation to local temperature affect immunity and fecundity in Drosophila melanogaster. PLoS Pathog. 4, e1000025

60 Boete, C. (2009) Anopheles mosquitoes: not just flying malaria vectors. . .especially in the field. Trends Parasitol. 25, 53-55

61 Macdonald, G. (1957) The Epidemiology and Control of Malaria, Oxford University Press

62 Smith, D.L. and McKenzie, F.E. (2004) Statics and dynamics of malaria infection in Anopheles mosquitoes. Malar. J. 3, 13

63 Chouaibou, M. et al. (2008) Dynamics of insecticide resistance in the malaria vector Anopheles gambiae s.l. from an area of extensive cotton cultivation in Northern Cameroon. Trop. Med. Int. Health 13, 476-486

64 Weill, M. et al. (2004) The unique mutation in ace-1 giving high insecticide resistance is easily detectable in mosquito vectors. Insect. Mol. Biol. 13, 1-7

65 Diabate, A. et al. (2004) The spread of the Leu-Phe kdr mutation through Anopheles gambiae complex in Burkina Faso: genetic introgression and de novo phenomena. Trop. Med. Int. Health 9, 1267-1273

66 Duron, O. et al. (2006) High Wolbachia density correlates with cost of infection for insecticide resistant Culex pipiens mosquitoes. Evolution 60, 303-314

67 McCarroll, L. and Hemingway, J. (2002) Can insecticide resistance status affect parasite transmission in mosquitoes? Insect. Biochem. Mol. Biol. 32, 1345-1351

68 Read, A.F. et al. (2009) How to make evolution-proof insecticides for malaria control. PLoS Biol. 7, e1000058

69 Hancock, P.A. (2009) Combining fungal biopesticides and insecticidetreated bednets to enhance malaria control. PLoS Comput. Biol. 5, e1000525 